\title{
Search for p-mode Frequencies on Procyon A
}

\author{
M. Martic, J. C. Lebrun, J. Schmitt and J.-L. Bertaux \\ Service d'Aéronomie du CNRS, BP No 3, 91371, Verrières le Buisson, \\ France
}

\begin{abstract}
Following the recent evidence for the presence of an excess of power around $1 \mathrm{mHz}$ in the frequency spectrum of the Doppler shift measurements for Procyon (Martic et al., 1999), we searched for individual frequencies of p-modes from three independent observing runs $(5$, 10 and 15 nights). All observations (December 1997, November 1998, January 1999) were made with the ELODIE spectrograph on the $1.93 \mathrm{~m}$ telescope at Observatoire de Haute Provence. The individual peaks in cleaned power spectra of each time series in the interval of excess power are compared with the predicted $\mathrm{p}$-mode frequencies from stellar models (Chaboyer et al., 1999) for Procyon A.
\end{abstract}

\section{Introduction}

Precise measurements of the frequencies and amplitudes of the solar oscillations (e.g. by integrated sunlight observations from the SOHO mission, see this proceeding) have led to detailed inferences about the solar internal structure. Because of the wealth of knowledge obtained from helioseismology there is a strong impetus to use the pulsation analysis techniques to probe the interiors of the solar-like stars. From the ground the application of the seismic techniques to these stars is difficult because of extremely small variations in intensity and velocity associated with $\mathrm{p}$-modes and need to have adequate temporal coverage to resolve the modes. The best prospects for observing $\mathrm{p}$-mode oscillations in individual solar-type stars is offered by spectroscopic Doppler measurements. Recently, we have found the excess power in the frequency spectrum of the Doppler shift measurements for Procyon A (Martic et al., 1999). Previous attempts to detect the signatures of the p-modes and their characteristics (e.g. large splitting) on Procyon (Gelly et al. 1986, Bedford et al., 1995, Brown et al., 1991 etc.) were not conclusive or not confirmed by later observations by the same authors. Some of them were maybe biased by previous uncertainty of the estimates of Procyon mass. Here we report the first results of the search for p-modes in Procyon data taken between 1997 and 1999.

\section{Observations}

The observations were carried out with the ELODIE spectrograph on the $1.93 \mathrm{~m}$ OHP Telescope. The ELODIE is fiber-fed cross-dispersed echelle spectrograph that is optimised for precise stable Doppler measurements (Baranne et al., 1996). 

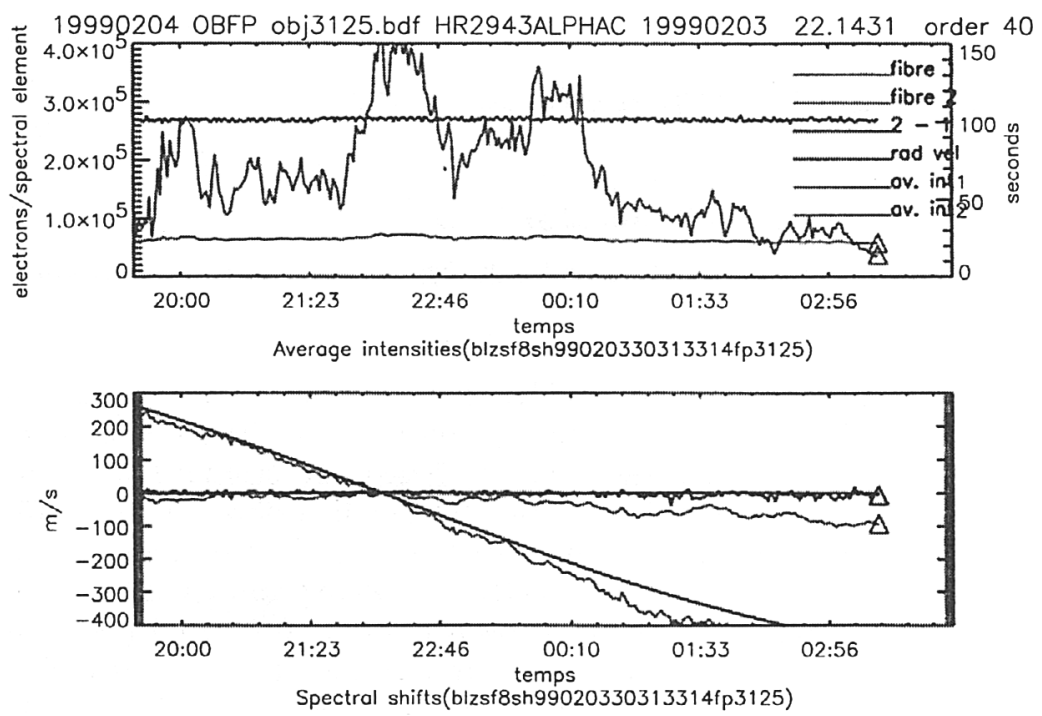

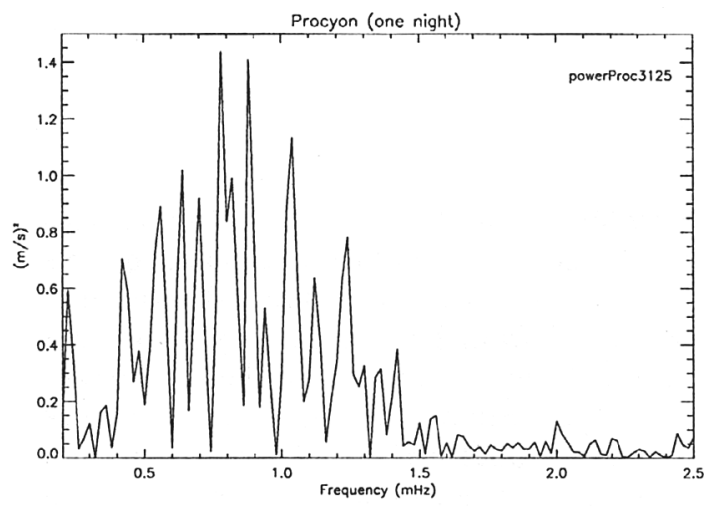

Figure 1. Example of the real-time display of the Procyon observations over night: Average intensity for one order from 40 s exposures; corresponding Doppler shift superposed to the radial earth velocity and instrumental shift as measured by the Fabry-Perot spectrum; Power spectrum of the weighted mean (40 orders) Doppler shift, computed with $40 \mu \mathrm{Hz}$ resolution.

In asteroseismic configuration, as described in Martic et al. (1999), we record simultaneously with stellar spectrum the closely-spaced channelled spectrum from a fixed Fabry-Perot (FP) interferometer in order to monitor the spectrograph drifts, with a high precision, for very short exposure times (30 to $40 \mathrm{~s}$ ) of bright objects, like Procyon $\left(m_{\mathrm{v}}=0.34\right)$. The real-time extraction of the spectra (67 orders) for both star and FP and computation of the velocity shifts take less than $40 \mathrm{~s}$, which is of the order of the CCD readout time. In the Fig. 1 we show the Doppler shift measurements for one of the best nights (high $\mathrm{S} / \mathrm{N}$, low rms residual) of Procyon observations. The corresponding power spectrum of the weighted mean Doppler shift over 40 orders is shown on the lower part of the Fig. 1. From three observing runs in 1997, 1998 and 1999 we selected respectively, 1047, 2011 and 2207 spectra for further power spectra analysis. 

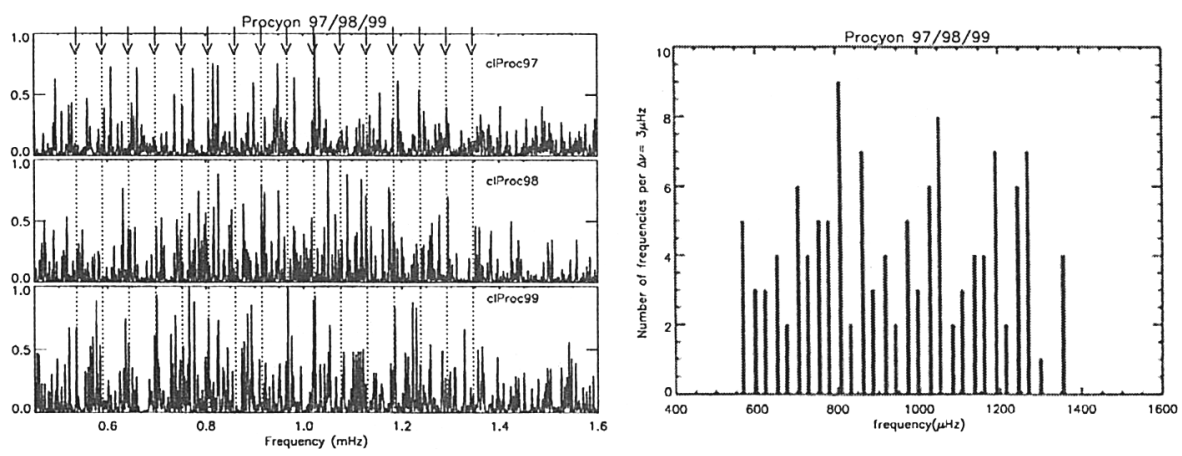

Figure 2. Cleaned power spectra of the independent time series and the histogram of the major peaks in the interval of excess power.

\section{Results}

The analysis of the time series of Doppler shift measurements obtained from three independent observing runs between 1997 and 1999 confirmed the presence of a strong concentration of power between $0.5 \mathrm{mHz}$ and $1.5 \mathrm{mHz}$, which is likely to be due to solar-like p-modes on Procyon. The corresponding periodograms are presented in Martic et al. (2000). The rms scatter varies from 3 to $4 \mathrm{~ms}^{-1}$ depending on the sequence and the lowest mean white noise level deduced from the power spectrum for the longest sequence is $0.008\left(\mathrm{~ms}^{-1}\right)^{2}$. We checked the statistical significance of the peaks in the range of the excess power and confirmed the estimation of the amplitude of the oscillations of the order of $50-70 \mathrm{cms}^{-1}$, which is below predicted amplitudes by Houdek et al. (1999). The irregular shape of the excess power can be explained by the interaction of modes with different phases through observational window and interference with randomly distributed noise peaks (see simulations in Martic et al., 1999, Barban et al., 1999). The identification of individual p-mode frequencies is difficult because of these interferences and daily gaps which suppress and/or reinforce peaks by mixing multiple frequencies in power spectra.

In order to reduce the effects of the window functions from our singlesite measurements we processed the times series with the CLEAN procedure (Roberts et al. 1987). Fig. 2 shows the results of the cleaning for three complete sequences from 1997, 1998 and 1999 runs. Several peaks seem to be recurrent within $2 \mu \mathrm{Hz}$ resolution, and consistent with theoretical frequencies predicted from the standard model (Chaboyer et al., 1999). Some large peaks correspond to the sidelobes at $\pm 11.6 \mu \mathrm{Hz}$, chosen by CLEAN procedure instead of the central peaks seen in power spectra. For further statistical analysis we used several independent three-nights data segments, where CLEAN seemed to work best. The histogram of the major peaks in the range of predicted frequencies $(n=$ $[9,24]$ and $l=[0,1])$ is shown on the right part of the Fig. 2 .

Finally, in the Fig. 3 we display the first echelle diagram of Procyon oscillations from the 1999 observing run. It was constructed from the CLEANed spectrum by adding the values over threshold for the frequencies modulo $\Delta \nu_{0}=$ $54 \mu \mathrm{Hz}$, within cells of $1.5 \mu \mathrm{Hz}$ (frequency bin is $0.5 \mu \mathrm{Hz}$ ). The threshold is de- 


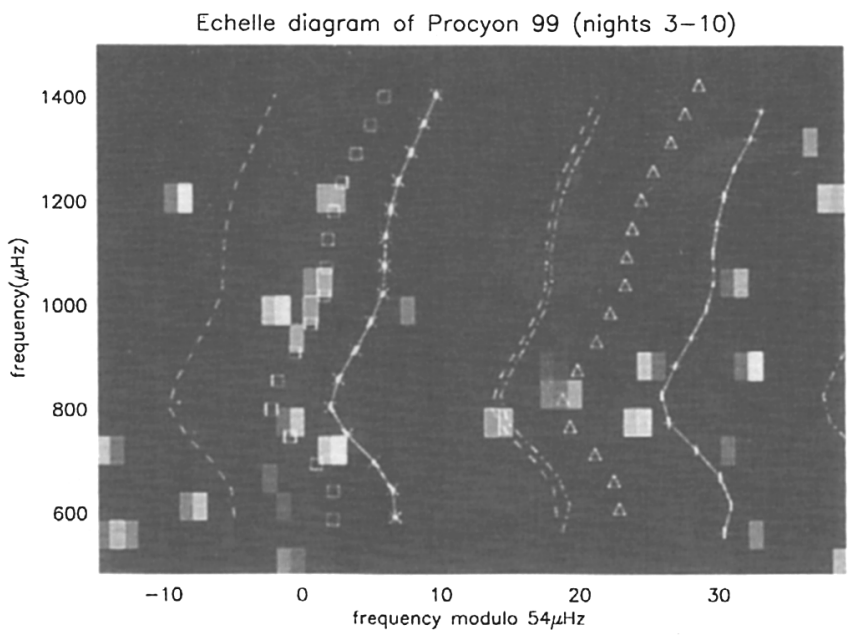

Figure 3. Echelle diagram of the Procyon power spectrum, modulo $54 \mu \mathrm{Hz}$. Theoretical frequencies from the standard model are indicated by asterisk, plus, square and triangle respectively for $l=0,1,2$ and 3 .

termined by the mean noise level of the time sequence. The echelle diagram from Procyon observations reveals the overall distribution of the predicted pmodes from Chaboyer et al. (1999). Taking account the frequency resolution of $1.5 \mu \mathrm{Hz}$, the average large splitting $\Delta \nu_{0}$ is of the order of $54 \mu \mathrm{Hz}$ which is in a good agreement with the theoretical predictions.

A detailed statistical analysis and the identification of the p-mode frequencies from different time series of Procyon Doppler measurements will be presented in the next paper.

Acknowledgments. We are grateful to OHP staff for the on site support of the observations. We thank A. Baglin, C. Barban and E. Michel for their contribution in obtaining Procyon results.

\section{References}

Baranne, A., Queloz, D., Mayor, M. et al., 1996, A\&AS, 119, 373

Barban, C., Michel, E., Martic, M. et al., 1999, A\&A, 350, 617

Bedford, D.K., Chaplin, W.J., Coates, A.R. et al., 1995, MNRAS, 273, 367

Brown, T.M., Gilliland, R.L. et al., 1991, ApJ, 368, 599

Chaboyer, B., Demarque, P. and Guenther, D.B., 1999, ApJ, 525L, 41

Gelly, B., Grec, G., Fossat, E., 1986, A\&A, 164, 383

Houdek, G., Balmforth, N.J. et al., 1999, A\&A, 351,582

Martic, M., Schmitt, J., Lebrun, J.C. et al., 1999, A\&A, 351, 993

Martic, M., Lebrun, J.C., Schmitt, J. et al., SOHO-10/GONG-2000, in press

Roberts, D.H., Léhar, J. and Dreher, J.W., 1987, AJ, 93(4), 968 